\title{
Pratiques
}

Linguistique, littérature, didactique

187-188 | 2020

Enseignement du texte littéraire dans l'espace

francophone: pratiques, formation, recherche

\section{Les forums scolaires de lecteurs au service des approches analytiques des textes littéraires : intérêts et limites}

Educational forums of readers for analytical approaches to literary texts :

advantages and limitations

\section{Pierre Moinard}

\section{(2) OpenEdition}

Journals

Édition électronique

URL : https://journals.openedition.org/pratiques/9072

DOI : $10.4000 /$ pratiques.9072

ISSN : 2425-2042

Éditeur

Centre de recherche sur les médiations (CREM)

Référence électronique

Pierre Moinard, «Les forums scolaires de lecteurs au service des approches analytiques des textes littéraires : intérêts et limites », Pratiques [En ligne], 187-188| 2020, mis en ligne le 16 décembre 2020, consulté le 21 juillet 2021. URL : http://journals.openedition.org/pratiques/9072 ; DOI : https://doi.org/ 10.4000/pratiques.9072

Ce document a été généré automatiquement le 21 juillet 2021.

(c) Tous droits réservés 


\title{
Les forums scolaires de lecteurs au service des approches analytiques des textes littéraires : intérêts et limites
}

\author{
Educational forums of readers for analytical approaches to literary texts : \\ advantages and limitations
}

Pierre Moinard

\section{Introduction}

Considérer à priori les utilisations scolaires de forums de lecteurs comme des innovations didactiques reviendrait à présupposer qu'une technologie a le pouvoir de modifier par elle-même des choix d'enseignants comme certaines attitudes des élèves face à la lecture scolaire de textes littéraires. Or il est concevable que l'introduction de moments d'échanges en ligne au cours d'approches analytiques des textes littéraires laisse apparaitre des phénomènes de sédimentation ${ }^{1}$ qui conduisent à privilégier les démarches de lecture descendantes (Dufays, 2017). Dans deux contributions récentes, nous avons examiné des utilisations d'outils de communication asynchrone pour entrer dans des œuvres ou des textes narratifs (Moinard, 2019) et la diversification des écrits métatextuels dans des situations hybrides ${ }^{2}$ (Moinard, 2017). Dans cet article, nous interrogeons les intérêts et les limites de quelques usages de forums scolaires de lecteurs au service d'analyses d'extraits poétiques dans des classes de $3^{\mathrm{e}}, 2^{\text {nde }}$ et $1^{\text {re3 }}$. Parce qu'ils cristallisent des interactions écrites entre les apprentis lecteurs et leurs professeurs à différents moments des séances, les forums offrent des occasions d'examiner les évolutions des lectures des élèves comme les choix des enseignants expérimentateurs. Nous cherchons à savoir dans quelle mesure les utilisations des forums conduisent à une diversification de propositions interprétatives et amènent les 
élèves à questionner eux-mêmes les textes. Conjointement, nous interrogeons les conditions favorables ou non à l'effort interprétatif.

2 Des réponses à ces questions sont apportées par des analyses de trois séances intégrant des échanges en forums de lecteurs dans des établissements sociologiquement variés de l'académie de Versailles en 2015 et 2016. Les professeurs ont conçu leurs enseignements sans avoir été informés d'un scenario pédagogique attendu. La première section de l'article apportera quelques précisions sur les situations examinées avant d'exposer le cadre théorico-méthodologique et d'avancer deux hypothèses de recherche. Les cheminements interprétatifs exprimés dans les fils de billets seront décrits dans une deuxième section. La troisième section reviendra sur la tentation d'un contrôle des lectures manifestée dans certains usages de forums. La dernière section rendra compte de tentatives pour relancer des analyses interprétatives lors des moments de relecture collective de billets.

\section{Situations étudiées. Cadre théorique et méthodologique}

3 Tout d'abord nous précisons les caractéristiques des trois séances hybrides étudiées dans cet article.

4 La situation 1 réunit des élèves de $3^{\mathrm{e}}$, issus de catégories socio-professionnelles défavorisées, lors d'une séance en deux temps consacrée à la lecture analytique ${ }^{4}$ d'un extrait de Cahier d'un retour au pays natal d'Aimé Césaire dans le cadre d'une séquence sur la littérature engagée. L'extrait de 25 lignes comprend 14 versets évoquant la cale du navire négrier et la condition des esclaves (de « Les Blancs disent » à « la menace de ses grondements intestins $»^{5}$ ).

5 La situation 2 développe une étude du poème de V. Hugo «Sultan Mourad » dans son intégralité, en seconde dans un lycée accueillant des élèves des classes moyennes. Le travail s'inscrit dans une séquence regroupant des poèmes de La Légende des siècles et des Châtiments.

6 Dans la situation 3, la lecture analytique ${ }^{6}$ du poème de P. Éluard, « Notre vie » en classe de première technologique est orientée par la production collaborative d'un commentaire littéraire. Les élèves de première technologique d'un lycée recrutant dans des milieux plutôt défavorisés sont invités à co-construire progressivement en ligne le « commentaire de la classe ».

7 Le tableau suivant précise les différentes consignes délivrées pour les échanges en ligne et les corpus analysés.

Tableau 1. Corpus analysés et consignes pour les échanges en ligne

\begin{tabular}{|l|l|l|l|}
\hline $\begin{array}{l}\text { Classe et texte } \\
\text { étudié }\end{array}$ & $\begin{array}{l}\text { Échanges en ligne (consignes; nombre de billets; durée } \\
\text { des échanges) }\end{array}$ & Captations vidéo \\
\hline
\end{tabular}




\begin{tabular}{|c|c|c|}
\hline $\begin{array}{l}\text { Situation } 1 \text { : } \\
3^{\mathrm{e} ;} \text { Aimé Césaire, } \\
\text { Cahier d'un retour } \\
\text { au pays natal, } 1947 .\end{array}$ & $\begin{array}{l}\text { Sur deux murs virtuels Padlet configurés en forum } \\
\text { "Si vous deviez associer ce poème à un sport, quel sport } \\
\text { choisiriez-vous? Pour quelles raisons? Vous justifierez } \\
\text { votre réponse en vous appuyant sur des citations précises } \\
\text { et analysées." } \\
19 \text { contributions les } 14 \text { et } 15 \text { janvier } 2016 . \\
\text { "Lors de la dernière séance [...] sur le texte "Les Blancs } \\
\text { disent ..." d'Aimé Césaire, l'un d'entre vous a souligné un } \\
\text { problème à propos de la citation suivante et notamment } \\
\text { du choix du mot "ténia" pour qualifier les esclaves: } \\
\text { "L'affreux ténia de sa cargaison ronge les boyaux fétides } \\
\text { de l'étrange nourrisson des mers !" (l. 21-22). Quelle } \\
\text { image cela donne-t-il des esclaves? Selon vous, pourquoi } \\
\text { l'auteur associe-t-il les esclaves au "ténia"? Que veut-il } \\
\text { signifier ici? Vous développerez votre réponse en } \\
\text { n'hésitant pas à vous appuyer sur des citations. } \\
19 \text { contributions du } 22 \text { au } 31 \text { janvier } 2016 \text {. }\end{array}$ & $\begin{array}{l}\text { Autocaptation de } \\
\text { deux heures le } \\
14 \text { janvier 2016; } \\
\text { captation par le } \\
\text { chercheur le } \\
15 \text { janvier 2016. }\end{array}$ \\
\hline $\begin{array}{l}\text { Situation } 2 \text { : } \\
2^{\text {nde }} \text {; Victor Hugo } \\
\text { "Sultan Mourad ", } \\
\text { La légende des } \\
\text { Siècles, } 1859 .\end{array}$ & $\begin{array}{l}\text { Découverte du poème en autonomie; avant le travail en } \\
\text { classe. Sur un forum de lecteurs commun à deux classes } \\
\text { de seconde. } \\
\text { "Conseilleriez-vous la lecture de ce poème à un ami? } \\
\text { Oui/non? Pourquoi ? } 3 \text { arguments à l'appui. » } \\
21 \text { billets du } 18 \text { octobre au } 1^{\text {er }} \text { novembre } 2015 \text {. }\end{array}$ & $\begin{array}{l}\text { Captation de deux } \\
\text { heures de cours } \\
\text { par le chercheur, } \\
\text { le } 2 \text { novembre } \\
2015 .\end{array}$ \\
\hline $\begin{array}{l}\text { Situation 3: } \\
1^{\text {re }} \text { technologique; } \\
\text { Paul Éluard } \\
\text { « Notre vie ", } 1947 .\end{array}$ & 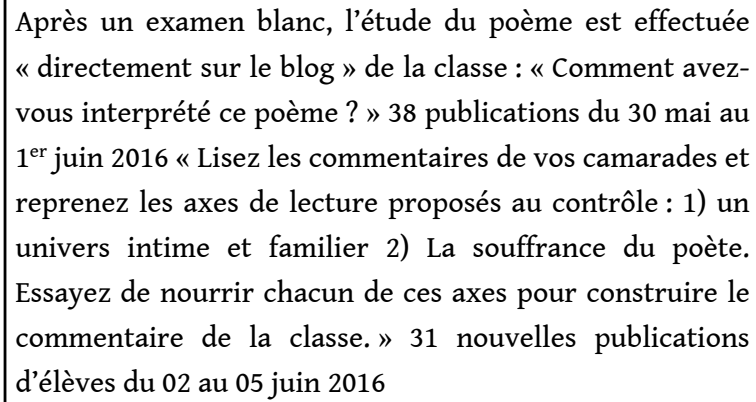 & $\begin{array}{l}\text { Sans captation } \\
\text { associée }\end{array}$ \\
\hline
\end{tabular}

8 Deux outils de communication asynchrone différents ont été utilisés : un blog de classe présentant une rubrique configurée en forum (situations 2 et 3 ) ou des murs virtuels Padlet, eux aussi configurés en forum ${ }^{7}$ (situation 1). Pour chaque situation, ont également été analysés des entretiens semi-directifs avec les professeurs et avec six élèves aux profils différents.

Les échanges asynchrones sont déterminés par des conditions d'utilisation des forums propres à chaque situation. La nature et les évolutions des écrits asynchrones ne dépendent pas uniquement de fonctionnalités techniques ${ }^{8}$. Elles sont largement déterminées par les consignes professorales et les explications dont elles font l'objet. En outre, l'archivage public des billets révèle les manières dont les tâches demandées sont investies; les séquences de billets forment un co-texte orientant les contributions ultérieures. En retour, la visibilité des lectures des élèves apportée par les fils de discussion peut provoquer différentes adaptations chez les professeurs. 

les expériences spontanées de lecture, à l'instar d'autres formes d'écritures de la réception (Le Goff \& Fourtanier, 2017). De surcroit, dans ces billets, les retours sur les lectures et les textes peuvent constituer des actes de langage. Dans l'exemple suivant pris dans la situation 3, le post avance une proposition en attente de confirmation: « La phrase "donner la vie" au vers 7 prend un sens étrange car elle crée un contraste avec la mort » (Gabrielle, 12). Le jugement d'étrangeté contribue à identifier un effet tout en posant une demande indirecte d'éclaircissements à destination des pairs et de la professeure.

11 Nous avons choisi de rendre compte d'études de textes poétiques présentant une dimension narrative marquée. Ces extraits permettent donc des retours sur les logiques d'action ou des intrigues comme les remarques sur la polysémie des images ou la perception de configurations sonores.

12 Afin de rendre compte de la diversité des postures de lecture exprimées à travers des écrits interactifs de lecteurs et en classe, le cadre théorique de la recherche intègre la notion d'» activité fictionnalisante du sujet lecteur» (Langlade, 2008) au paradigme dialectique de la «lecture littéraire» (Dufays, Gemenne \& Ledur, 2015 [1996]). Ce paradigme envisage l'alternance de deux modalités complémentaires de lecture, la " participation » centrée sur les représentations mentales du lecteur (ses imaginations et émotions) et la «distanciation» qui correspond qui correspond à la fois à l'interprétation du texte et à «la réflexivité du lecteur dans l'activité singulière du "sujet lecteur" ». (Sauvaire \& Dufays, 2020, p. 239). Les « activités fictionnalisantes du sujet lecteur » que définit G. Langlade (2008) distinguent précisément trois aspects de la «lecture participative»:

- la simulation mentale de perceptions et d'émotions de personnages (aspect imaginaire et empathique);

- les jugements moraux sur les personnages ou les situations (aspect axiologique) ;

- et les appréciations de choix d'écriture (aspect esthétique).

13 La variété des retours asynchrones sur les expériences de réception nous conduit à préciser encore la définition de la participation ${ }^{9}$ du lecteur. À partir de travaux de J.M. Schaeffer (2015) et de M. Macé (2011), nous proposons d'envisager la participation comme une conduite autorégulée. Le maintien d'une lecture (pleinement) participative en contexte scolaire peut se concevoir comme une tâche mobilisant deux régimes attentionnels du lecteur à l'égard de ses propres réactions. D'une part, le lecteur isole (par une attention sélective et focalisée) des réactions bien identifiables et signifiantes à ses yeux. Ainsi fonde-t-il des jugements empathiques, axiologiques ou esthétiques (y compris des jugements d'étrangeté). D'autre part, le lecteur peut se mettre aux aguets de vécus subjectifs diffus ou peu caractérisables en engageant une attention plus flottante. L'association de ces deux régimes attentionnels est appelée par la demande faite aux élèves de « réagir » en lecteurs en dépit de l'altérité des textes patrimoniaux.

Concevoir la participation comme une conduite justifie de prêter intérêt aux efforts de lecteurs pour s'immerger dans des textes à partir de témoignages d'une participation incomplète. À propos du texte poétique, A. Rodriguez (2013, p. 88) distingue «quatre issues de l'acte de lire et d'entrer dans un texte. La catégorisation qu'il expose dans les lignes suivantes présuppose une conduite participative :

«j'ai lu l'ouvrage et je suis entré dans ce monde » (réalisation satisfaisante), «j'ai lu

l'ouvrage mais je ne suis pas entré dans ce monde » (réalisation insatisfaisante), «je 
n'ai pas fini l'ouvrage mais je suis entré dans son monde" (interruption satisfaisante), « j'en ai assez lu cela ne mène à rien » (interruption insatisfaisante). (ibid.) lecteurs des manifestations d'un effort de participation en lien avec le travail interprétatif. Pour analyser les écrits asynchrones revenant sur les expériences de lecture, nous avons défini les trois catégories et deux sous-catégories suivantes. Les écrits reconstituant une expérience du lecteur sont appelés des "textes de lecteurs participatifs ». Une première sous-catégorie réunit les expressions directes ou imagées des vécus subjectifs, comme dans le billet suivant revenant sur la lecture de «Sultan Mourad» :

Ce texte est assez difficile à comprendre car il passe de la mort d'un porc aux phénomènes naturels de la Terre comme le tonnerre, les tourbillons, la brume... J'ai pas apprécié lire ce poème car je n'y comprends pas grand-chose alors je ne le conseillerai pas à un de mes amis. (Julie, $5^{13}$ )

17 En réponse à la consigne rappelée dans le tableau 1, ce texte de lecteur participatif témoigne d'une « réalisation insatisfaisante». Le contributeur souligne ce qui dans le poème déjoue des attentes de cohérence textuelle («il passe de ...à... »). Ainsi le billet revient-il à la fois sur un horizon d'attente propre à la communauté interprétative des élèves et sur l'effort pour s'approprier un texte " assez difficile à comprendre " parce que rendant l'immersion malaisée.

18 Une seconde sous-catégorie des retours sur la lecture participative renvoie à une centration sur le texte; elle réunit des paraphrases ciblées et des citations dans un effort pour isoler précisément ce qui déclenche des réactions. Dans le post suivant par exemple, les réactions de la lectrice de «Notre vie» ne sont pas caractérisées directement :

Dans ce poème, la mort s'empare du personnage comme dans un livre ouvert : « Et

la mort entre en moi comme dans un moulin » La mort l'entoure et reste près de lui.

(Céline, 11)

19 La lectrice exprime sa méditation sur une image à travers une paraphrase créative : elle reformule une expression du poète (« la mort s'empare du personnage ») et la prolonge (" comme dans un livre ouvert »).

Enfin, nous appelons "synthèses de la participation et de la distanciation", une dernière catégorie d'analyse, qui regroupe des énoncés thématisant des liens entre les modalités de lecture. Plus réflexives que les seules mentions de réactions, ces synthèses 
témoignent d'un effort d'interprétation prenant appui sur la clarification de l'expérience participative. C'est le cas dans ce billet commentant également «Notre Vie $»$ :

Dès le premier vers, l'auteur [...] dit que sa vie est ensevelie c'est-à-dire que la vie qu'il menait avec sa femme est finie mais aussi profondément enfouie en lui au point de le plonger progressivement dans un mutisme « Mon passé se dissout je fais place au silence ». (Sophie, 19)

21 À l'instar de l'exemple précédent, la réflexion sur les résistances du poème se traduit par une paraphrase (« l'auteur dit que »; «c'est-à-dire que »). Mais ici, la contributrice reprend trois fois l'isotopie de l'ensevelissement, l'applique à des actants différents pour avancer une signification: "sa vie est ensevelie " puis «la vie qu'il menait est enfouie en lui » enfin « au point de le plonger progressivement dans ». Le poète luimême est vu comme un corps enseveli dans un renversement qui interroge le « mutisme » paradoxal du sujet lyrique. La citation à la fin du message invite les autres contributeurs à sonder encore les significations.

La plupart des synthèses de la participation et de la distanciation relevées dans les situations étudiées proposent des interprétations actualisantes selon la définition qu'en donne Y. Citton (2007) :

Une interprétation littéraire d'un texte ancien est actualisante dès lors que a) elle s'attache à exploiter les virtualités connotatives des signes de ce texte, b) afin d'en tirer une modélisation capable de reconfigurer un problème propre à la situation historique de l'interprète, $\mathrm{c}$ ) sans viser à correspondre à la réalité historique de l'auteur, mais d) en exploitant, lorsque cela est possible, la différence entre les deux époques (leur langue, leur outillage mental, leurs situations socio-politiques) pour apporter un éclairage dépaysant sur le présent (ibid., p. 265).

Notre catégorisation ${ }^{14}$ des divers retours sur les textes littéraires permet de suivre des évolutions des écrits en ligne (et des paroles échangées en classe). La démarche d'analyse des fils consiste à identifier des cheminements réflexifs après avoir catégorisé les remarques échangées lors des interactions asynchrones (et synchrones) et comparer leurs contenus. L'analyste peut alors se montrer attentif aux efforts des élèves pour maintenir une lecture participative en contexte scolaire en relation avec le travail d'analyse interprétative.

Les unités d'analyses que nous retenons permettent d'envisager deux hypothèses quant aux effets des séances hybrides sur la formation des lecteurs. D'une part, nous conjecturons que les consignes d'écriture asynchrones et les manières d'accompagner les travaux en ligne favorisent les prises de recul sur les expériences de lecture et l'émergence d'interprétations. D'autre part, nous attendons que les retours en classe sur des billets conduisent à relancer l'effort interprétatif des élèves.

\section{Une relative diversification des retours sur les réceptions à travers leur partage}

Les analyses montrent une certaine réussite des échanges asynchrones sur le plan de la communication. Les professeurs observés ont en général su faire dévolution ${ }^{15}$ (Brousseau, 1998) d'un partage de retours sur des expériences de lecture. Ils y sont parvenus en proposant des consignes qui invitent les élèves à exprimer des réactions personnelles et leur permettent d'exprimer des difficultés à accéder à une lecture participative de textes littéraires. En outre, les enseignants ont évité d'évaluer 
sommativement les écrits en ligne pour favoriser la collaboration. Aussi ont-ils choisi de limiter les conseils rhétoriques qui, à priori, risquaient de formater les billets.

Ces usages du forum entrainent le plus souvent un enrichissement des écrits partagés par les élèves lecteurs. Les enchainements de paraphrases sélectives du texte lu et de descriptions d'expériences de lecture aboutissent parfois à des propositions interprétatives actualisantes. Cependant la diversification des significations et l'accès à des questionnements sur les textes sont souvent menacés par l'effort même que consentent les contributeurs pour maintenir la communication à distance.

Les échanges asynchrones entre élèves lecteurs montrent en effet une tendance aux répétitions en écho malgré des demandes explicites de discussion par les professeurs. L'analyse du fil «Sultan Mourad» montre des limites à la diversification des lectures exprimées en ligne en réponse à la consigne: "Conseilleriez-vous la lecture de ce poème à un ami ? Oui/Non? Pourquoi?». Pour autant, cette demande de recommandation, qui transpose une pratique sociale ${ }^{16}$, invite efficacement au partage des jugements de gout ou de valeurs en communauté d'élèves lecteurs.

Dès le premier billet, trois justifications d'une lecture dysphorique sont fortement affirmées: la représentation hyperbolique des atrocités du sultan; la rupture de cohérence mimétique introduite par le geste de compassion inexplicable de Mourad envers un pourceau à l'agonie dans Bagdad $^{17}$; enfin la rupture de cohérence axiologique introduite par la rédemption finale du $\operatorname{sultan}^{18}$. Le développement des textes de lecteur participatif présente trois cheminements. D'abord, les billets en écho reconstituent de plus en plus précisément des jugements axiologiques, comme dans ce billet :

Le sultan Mourad est pour moi un réel meurtrier, il est pourtant décrit comme puissant, vainqueur et maitre du Monde, cela m'agace donc. (Victoria, 10)

Les contributions qui exposent les entraves à la participation apparaissent un peu plus tard. Le billet 6 par exemple précise que l'histoire du sultan n'est "pas ordonnée » (Emma). Le billet 9 prolonge et précise la dénonciation des étrangetés du récit poétique :

Je trouve qu'il n'y a pas assez de détails contrairement aux histoires de maintenant. $[. .$.$] je pense que ce poème n'est pas fait pour des gens de notre époque et encore$ moins pour des personnes de notre âge, que ce soit le contexte de l'histoire ou encore la façon dont il est écrit. Il est destiné aux personnes vivant à la période où a été écrit le poème (Léa, 9).

Dans ce billet fortement réflexif, la contributrice s'étonne de la gestion des informations narratives dans le poème avant de thématiser un sentiment de décalage avec l'horizon d'attente inscrit dans le poème. Il est intéressant de constater que la reconstitution fine de son expérience d'élève lectrice conduit Léa à poser l'hypothèse d'une conduite immersive à laquelle elle n'accède pas. Enfin certains lecteurs scripteurs explorent des facettes différentes de la participation en mettant en tension les jugements axiologiques partagés et des appréciations esthétiques de l'écriture poétique comme dans ces deux exemples :

Certains passages m'ont paru plus clairs que d'autres; par contre je trouve certaines rimes et vers, très éloquents et marquants. (Emma, 6)

Le sens du texte reste flou, complexe et peu compréhensible. Cependant les rimes présentes en fin de lignes apportent un côté harmonieux et très poétique au texte. (Orlane, 8) 
31

billets complexifient les reconstitutions des expériences centrées sur pour interroger la légitimité d'un jugement exclusivement axiologique (sur le personnage éponyme). Seul le dernier billet avance une synthèse de la participation et de la distanciation interprétative :

Je recommanderais la lecture de ce texte à un ami car malgré la complexité de ce texte je le trouve intéressant, plus précisément je trouve la fin de ce poème intéressante car ce poème dit qu'un homme en l'occurrence le Sultan Mourad dans ce texte peut commettre les pires crimes du monde mais seulement faire une bonne action dans sa vie pour aller au paradis. (Clément, 22)

Clément est le seul élève qui s'autorise à marquer une différence d'avis en situant sa lecture par rapport à celles des autres («malgré la complexité de ce texte je le trouve intéressant»). Sa proposition se distingue par une centration sur le pôle artistique qui permet d'avancer une hypothèse d'intentionnalité (« ce poème dit que... »).

des 22 billets ne conduit donc pas à des échanges ouvertement contradictoires parce que le jeu de reprises enrichissantes qui le structure privilégie les ajouts aux expressions de désaccords. Le partage de réactions consensuelles aide à maintenir la communication en différé sans relancer le questionnement sur des significations du poème. Le dispositif favorise surtout la description (parfois fine ) des difficultés posées par la tâche de lecture impliquée d'un poème patrimonial. Trois facteurs ${ }^{19}$ réunis dans la situation 2 peuvent limiter l'exploration des potentialités signifiantes du poème. D'abord, la consigne précédemment rappelée invite à défendre des positions tranchées non à discuter des jugements. Ensuite, il est possible que la situation d'échange sur le forum ait accentué une influence des premiers billets exposant les obstacles à l'entrée dans le poème sans interroger des significations du texte étudié ${ }^{20}$. Enfin, la professeure a habitude de prolonger les temps de partages des réactions de lecteurs ${ }^{21}$ lors des lectures analytiques.

ans les trois situations étudiées, les échanges asynchrones manifestent peu de désaccords explicites entre élèves lecteurs. Ce constat, qui a souvent surpris et déçu les professeurs, traduit un besoin partagé par les contributeurs de maintenir l'aménité des échanges à distance. Ainsi plusieurs contributrices au fil « Notre vie » choisissent de souligner les convergences comme pour rassurer leurs camarades et s'inscrire dans un groupe soudé : la formule «je suis d'accord avec ... » est reprise six fois dans les 38 premiers posts.

Pourtant les entretiens avec des élèves révèlent une forme de recherche des divergences au cours de la relecture du fil qui précède l'écriture d'un billet. Des élèves de différentes classes témoignent d'une intériorisation d'un dialogue avec les billets d'autrui. Océane, une élève de la classe de première technologique, explique en fin d'année scolaire :

10:45 Océane : avant [d'écrire sur le forum] je lis aussi les billets des autres [...] après j'essaie de trouver des idées supplémentaires que moi j'ai remarqué dans le texte $[. .$.

12:52 Océane : mais on a tous des idées un peu différentes/22 c'est pour ça que c'est intéressant de lire les billets des autres. (Entretien du 26 mai 2016).

L'intérêt pour les gouts des autres semble relever d'une éthique de l'accueil des opinions " on a tous des idées un peu ${ }^{23}$ différentes »). Pour autant cet intérêt pour les lectures d'autrui n'est pas mis en relation avec un effort pour interroger les 
potentialités signifiantes des textes. En outre pour Océane, le but des travaux en ligne reste la création d'un texte collectif de lecteurs :

11:38 Océane : comme ça tous les billets tous ensemble forment vraiment quelque chose de complet [...]

12:35 Océane : c'est comme si c'était un gros billet collectif et qu'on donnait tous notre petite participation. (ibid.)

Cette élève conçoit le partage comme une accumulation de contenus d'emblée complémentaires sans présupposer que des billets puissent se contredire et que leur relecture puisse la conduire à prendre position entre des positions difficiles à concilier.

Cécile-Aurore, une élève de $3^{\mathrm{e}}$ ayant collaboré à l'étude du texte d'Aimé Césaire, avance des raisons analogues d'identifier une diversité de lectures exprimées en ligne tout en ajoutant une intéressante préoccupation critique :

03:10 Cécile-Aurore : [...] en général j'ai pas vraiment le même point de vue que les autres et puis voir le leur ça/c'est une bonne chose pour se rendre compte de ce qu'on fait [...]

05:18 Le chercheur : d'accord/à quel moment lis-tu les billets des autres?

05:30 Cécile-Aurore : euh avant [d'écrire]/pour avoir un peu une idée et puis éviter les répétitions/et puis sinon après [avoir posté] aussi pour voir un peu ce que les autres ont mis et découvrir leurs points de vue aussi si je les/puisque en général avant je survole juste ce qu'ils ont dit. (Entretien du 3 juin 2016)

Cette habituée des forums de discussion thématique sur la toile décrit une procédure élaborée reposant sur une double lecture des billets d'autrui, une première fois pour situer son propos puis, après avoir posté, pour cartographier les différents points de vue exprimés en réaction au sien. Cette nouvelle lecture des posts plus précise et critique ("c'est une analyse quand j'ai le temps») nourrit un débat intériorisé à distance. La contributrice s'intéresse aux points de vue des «autres » pour se rendre consciente de son originalité ( en général j'ai pas vraiment le même point de vue que les autres ») et pour préciser sa propre réflexion sur les textes («se rendre compte de ce qu'on fait »). Ainsi évoque-t-elle un geste d'étude qui pourrait être diffusé par un pointage professoral de divergences exprimées dans les billets pour provoquer des échanges entre lecteurs interprètes.

Dans les situations dont nous rendons compte, les professeurs favorisent le maintien d'une communication asynchrone offrant aux élèves la possibilité d'enrichir des descriptions de lectures participatives qui amènent à des propositions interprétatives. Pourtant la diversification des lectures reste modeste et est due avant tout à l'investissement de quelques élèves soucieux de relancer la réflexion. Cette première limite à une pluralisation des lectures est liée à une atténuation des désaccords par l'écriture de billets cherchant à agréger plus qu'à discuter les différentes propositions cette règle tacite étant acceptée par la grande majorité des élèves. L'harmonisation rapide des lectures résulte aussi des consignes d'écriture en ligne. D'une part, celles-ci cherchent favoriser l'implication dans les textes à travers l'accueil de jugements (" conseilleriez-vous »), d'associations personnelles ("si vous deviez associer ce poème à un sport ») et de lectures actualisantes (« comment avez-vous interprété ce poème »). D'autre part, la plupart des demandes présupposent l'acceptabilité (ou une certaine neutralisation) des divergences qui pourraient s'exprimer en ligne. Les oppositions qu'elles pourraient appeler relèvent de différences de gout (entre « amis » lecteurs), de la liberté même des associations analogiques ou des interprétations actualisantes des élèves. 
Une autre forme de réduction du questionnement collaboratif des lecteurs réside dans une utilisation des forums pour amener les élèves à accepter la lecture attendue par l'enseignant.

\section{La tentation d'un contrôle des lectures}

Il arrive que les professeurs hésitent à prendre en compte les propositions en ligne qu'ils ont eux-mêmes suscitées pour relancer une réflexion interprétative en collaboration. Face à des remarques imprévues ${ }^{24}$, ils sont parfois tentés de simplement trier les billets en fonction d'une lecture attendue. Les analyses de l'étude d'un extrait du Cahier d'un retour au pays natal en classe de $3^{\mathrm{e}}$ (la situation 1 ) révèlent une telle hésitation.

Cette séance de lecture analytique repose sur la réitération d'une même consigne pour le travail en équipe dans la classe puis pour le travail à distance :

Si vous deviez associer ce poème à un sport, quel sport choisiriez-vous? Pour quelles raisons? Vous justifierez votre réponse en vous appuyant sur des citations précises et analysées. (Professeur, 1)

L'entrée analogique est inspirée des travaux de S. Ahr (2018) sur les carnets de lecteurs dont le professeur a connaissance. Le recours à l'analogie vise à «favorise[r] une lecture actualisante » (ibid., p. 76) et immersive tout en aidant les élèves à faire des liens entre le texte étudié et leurs propres références. Avant de proposer la consigne associative, le professeur a expliqué le sens dénoté de mots ou d'expressions après une lecture à haute voix de l'extrait. Il a élucidé des images résistantes (parce que denses et polysémiques) en les traduisant en langage courant. Dès le début de la séance, il a centré l'attention des jeunes lecteurs sur « le message » de l'auteur :

10:07 Professeur : donc là encore une fois il est tout à fait normal que certains passages ne soient pas très clairs [...] moi ce qui m'intéresse c'est que vous ayez perçu quel est le message que l'auteur veut faire passer/pour ça on va utiliser une consigne qu'on avait déjà utilisée/à propos des poèmes engagés [Projection de la page du tableau virtuel Padlet portant la consigne]. (Heure du 14 janvier 2016, fichier vidéo 1)

Le professeur affirme une intention (unique) de l'auteur déjà suggérée par le contexte didactique (une séquence portant sur les " poèmes engagés »). Ainsi pose-t-il lui-même un projet de lecture qui consiste à retrouver dans les phrases du poème la dénonciation de l'esclavagisme. Ce projet n'est pas co-élaboré ${ }^{25}$ et n'engage pas un questionnement du texte.

Le premier fil fait apparaitre trois publications plus nettement interprétatives que les autres. Deux billets en particulier prolongent l'isotopie du lancer (présente dans le poème ${ }^{26}$ ) pour envisager la création poétique elle-même comme une arme :

Le sport que j'ai choisi pour représenter le texte d'Aimé Césaire est le lancer de javelot car l'auteur rejette, en lançant, toute sa haine envers les maitres. (Mayssane, 10)

Le texte d'Aimé Césaire me fait penser au lancer de javelot car il fait référence à une "canne insipide" (l.11) ce qui fait penser au manche du javelot. Toute la colère envers l'esclavage, le racisme, les Blancs est jetée lors du lancer de javelot. (Séphora, 15) 
élève. Dans les dernières minutes de l'heure de cours du 15 janvier, Aymeric interroge directement l'intention du poète postulée par le professeur :

50:24 Aymeric : mais aussi l'idée du ténia ${ }^{27}$ ça montre aussi que c'est un parasite/et du coup que les esclaves ne peuvent pas vivre sans leurs maitres

50:31 Professeur: // alors la question/c'est une idée intéressante l'idée du ténia du parasite/effectivement comparer les Noirs à un parasite [...] (Heure du 15 janvier 2016)

\section{Le professeur reprend cette
sur un second mur virtuel}

Lors de la dernière séance [...] sur le texte «Les Blancs disent ... » d'Aimé Césaire, l'un d'entre vous a souligné un problème à propos de la citation suivante et notamment du choix du mot «ténia » pour qualifier les esclaves : «L'affreux ténia de sa cargaison ronge les boyaux fétides de l'étrange nourrisson des mers !» (1.21-22). Quelle image cela donne-t-il des esclaves? Selon vous, pourquoi l'auteur associe-t-il les esclaves au "ténia»? Que veut-il signifier ici? (Le professeur, 1)

La demande d'une lecture résolument attentive à la puissance d'une image conduit les élèves à proposer de nouvelles significations comme dans ce post : 
Les esclaves sont associés à des parasites car ils sont des éléments perturbateurs qui vont se libérer de leurs chaines, de l'esclavage. L'auteur a raison de les associer à des parasites. (Sathya, 6) révolte partant des profondeurs du système esclavagiste et « qui peut tout détruire à l'intérieur » (Elise, 16). Une seule contribution propose une signification divergente ; pour l'auteur du billet 18 (Quentin), le ténia traduit l'asservissement des esclaves : « Ils sont donc totalement dépendants de leurs maitres ». Bien que, dans ce cas, la consigne semble viser directement à ouvrir une discussion en ligne, un débat n'a pas lieu. Comme le professeur réénonce la question d'Aymeric (« l'un d'entre vous a souligné un problème »), les élèves ont tendance à concevoir leurs posts comme des réponses successives qui lui sont adressées. Le travail en ligne reproduit donc la communication assez verticale ${ }^{28}$ qui prévaut dans les phases de "cours dialogué ». En outre, la seule remarque postée par le professeur sur le second tableau virtuel ne vise pas à entretenir une discussion des interprètes :

Vous semblez d'accord pour dire que l'auteur associe les esclaves à un parasite. Mais vous ne répondez pas à la question suivante : pourquoi le fait-il ? Que pensezvous du fait d'associer les esclaves à des parasites compte tenu de la visée de l'auteur avec ce texte? (Le professeur, 5).

Il s'agit de susciter une explication de l'image pour confirmer le sens global déjà exposé et non de provoquer l'exploration de la richesse connotative de la métaphore. Le professeur ne revient pas sur la seule « visée de l'auteur » qu'il envisage depuis le début de la séance pour favoriser des lectures plus attentives au registre lyrique que le poème développe.

mme, la première hypothèse de la recherche est assez largement validée. En dépit d'une tendance aux reprises de lectures consensuelles par les contributeurs, des cheminements réflexifs sont repérables à travers les séquences des billets d'élèves lecteurs. Les analyses montrent aussi à quelles conditions les forums scolaires de lecteurs peuvent devenir des espaces de réflexion sur les lectures et sur les textes. Les consignes d'écriture asynchrone aidant aux expressions suggestives des lectures, l'accueil des propositions (même imprévues) au moins dans les premières phases de travail en ligne favorisent les efforts des scripteurs lecteurs pour donner sens à leurs réactions, avancer des jugements et des interprétations partageables.

Pour préciser dans quelle mesure l'hybridation peut servir des relances interprétatives lors des lectures analytiques, il reste à examiner les modalités de retours sur des échanges en ligne et leurs effets sur la réflexion collaborative des élèves. 


\section{Les retours sur des billets de lecteurs}

\section{aboutit à l'émergence des propositions interprétatives suivantes :}

30:36 Fanny : faut croire qu'il y a quand même une part de bonté dans tout être méchant.

35:09 Jade : ben la suite c'est peut-être un peu comme une moralité aussi parce qu'il [inaudible] que tout le monde peut avoir une seconde chance.

35:25 Enzo : quoi qu'on ait fait dans notre vie c'est toujours la dernière chose qu'on fait qu'on retient/que les gens retiennent. (Heures du 2 novembre 2015, fichier vidéo 1)

18 : 27 Daniel : juste je crois que Mourad il est mauvais à cause de son pouvoir parce qu'il a tué plein de gens parce qu'il avait du pouvoir [inaudible] le porc il avait pas de pouvoir (Heures du 2 novembre 2015, fichier vidéo 2)

Le retour sur les billets exprimant des lectures subjectives conduit à objectiver ${ }^{29}$ des réactions exprimées et à centrer les élèves (jusqu'alors surtout attentifs aux [dé]plaisirs de leurs réceptions) sur de possibles intentions textuelles. Ces derniers avancent alors deux hypothèses d'interprétation autour de la question de la rédemption ou de celle du pouvoir.

Dans la situation 3, le travail hybride d'analyse du poème de P. Eluard repose sur la succession de moments d'écriture en ligne de natures différentes ce qui conduit à ménager à chaque étape des retours réflexifs sur les séquences antérieures de billets. La séance progresse en trois temps à travers les publications d'écrits asynchrones de plus en plus composés. Chaque temps de travail est d'abord lancé depuis une salle équipée d'ordinateurs ${ }^{30}$ avant de se prolonger à partir des terminaux domestiques. La première phase répond à une demande directe d'interprétation à travers des écrits non normés. Lors de la deuxième phase, les élèves sont invités à relire les 37 billets de l'étape 1 pour enrichir les analyses et les organiser en un texte cohérent selon deux axes de lecture préalablement donnés ; la professeure demande :

Lisez les commentaires de vos camarades et reprenez les axes de lecture proposés au contrôle $e^{31}: 1$ ) un univers intime et familier, 2) la souffrance du poète. Essayez de nourrir chacun de ces axes pour construire le commentaire de la classe. (La professeure, 38)

La troisième phase qui conduit à mutualiser des commentaires littéraires structurés en deux niveaux hiérarchiques (les axes et les sous-parties) implique une nouvelle relecture fine et réflexive des propositions déjà publiées. La professeure intervient au cours des échanges en ligne en valorisant les idées intéressantes. Enfin elle clôt les 
échanges en institutionnalisant une "proposition de synthèse de toutes [les] remarques » (La professeure, 71) qu'elle a réalisée par montage d'extraits des billets des élèves.

Les premiers billets des élèves lecteurs reviennent sur le vers 10 du poème, « La mort visible boit et mange à mes dépens ». Les élèves y lisent une représentation de «la mort » comme un personnage destructeur vampirisant le poète qui les conduit à saisir différentes significations. Après différents billets paraphrastiques réutilisant l'allégorie, une contributrice nomme le procédé :

On note la personnification de la mort en un esprit qui détruit tout sur son passage on remarque que la mort [...] finit par épuiser le corps du personnage ainsi elle le détruit physiquement. (Gabrielle, 18)

Un peu plus tard encore, le billet suivant témoigne d'un effort de définition d'un effet textuel :

On a l'impression que le poète nous fait part de son expérience avec la mort mais aussi pour qu'on ressente de la compassion pour lui. (Stacey, 33)

La contributrice suggère par la subordonnée à valeur finale que la "compassion " éprouvée par les jeunes lecteurs est une réponse à une intention du poète (" pour qu'on ressente de la compassion »). Cette intention auctoriale est postulée et interrogée («on a l'impression que »). Le billet de Stacey avance une "présomption d'intentionnalité » (Compagnon, 1998, p. 107) à partir de la définition d'un effet digne d'être analysé. La construction collaborative en ligne d'un (unique) commentaire de la classe par réorganisation et enrichissements des contenus publiés présente donc des bénéfices. Pour autant, la division du travail d'écriture métatextuelle normée réserve à l'enseignante l'organisation du propos : il ne leur est pas demandé de collaborer à la définition des «axes » (libellés d'emblée pour faciliter le commentaire littéraire), les paragraphes et leur ordre sont définis par la professeure. Dans ce cadre, les élèves ne sont pas explicitement invités à revenir sur ce qui mérite d'être commenté ni encouragés à problématiser la lecture.

Dans les séances que nous avons présentées, l'apparition de questionnements sur les expériences esthétiques de lecture ou sur les textes dépend à la fois des tâches d'écriture en ligne et des manières de revenir sur des billets. Deux démarches distinctes caractérisent les retours sur les échanges en ligne. Dans certains cas, le professeur pointe lui-même ou fait relire par des volontaires quelques billets pour institutionnaliser directement leurs contenus Alors la relecture publique de billets (en général vidéoprojetés) sert à valider des éléments d'analyse des textes comme dans la situation 1 :

05:13 [Le mur Padlet est vidéoprojeté] Professeur : quelqu'un veut-il lire ce qu'il a proposé ? (Heure du 15 janvier 2016)

Et dans la situation 2 :

05:43 [Vidéoprojection du billet 19] Professeur: je vais vous montrer. (Heure du 2 novembre 2015, fichier vidéo 2)

La situation 3 relève d'une autre démarche qui vise les relectures réfléchies de séquences entières de posts: "Lisez les commentaires de vos camarades". L'organisation collaborative du commentaire littéraire "de la classe » implique un travail d'évaluation des propositions avancées lors de la première phase de travail ; les élèves sont alors conduits à prendre en compte les relations entre différentes lectures exprimées. 
71 En définitive, la seconde hypothèse que nous avons posée est invalidée. Les relectures collectives des billets montrent de forts risques de fléchissement réflexif surtout quand ces moments sont conduits dans le but de réunir des éléments d'analyses à institutionnaliser. Les retours sur les billets alors ne favorisent pas les confrontations de lectures divergentes ou les mises en relations entre les billets. Les analyses montrent cependant des essais pour déléguer aux élèves la responsabilité de confronter les billets et de faire un choix voire d'opérer une synthèse de billets.

L'étude des séances hybrides attentive à l'émergence de nouveaux gestes didactiques permet donc de penser qu'un accompagnement actif et ciblé des échanges asynchrones aiderait à engager ou à préparer des retours plus réflexifs sur les billets de lecteurs. Il s'agit d'éviter que des divergences fécondes que les forums permettent de recueillir passent inaperçues sous la pression de quelques lectures dominantes reprises en écho.

\section{Conclusion}

73 À condition de ne les confondre ni avec des réseaux sociaux miniaturisés ni avec une classe numérique, les forums scolaires de lecteurs peuvent offrir des moyens d'accompagner les découvertes des textes littéraires comme le développement d'analyses interprétatives. Cependant, l'examen de pratiques hybrides indique que la visibilité de lectures d'élèves permise par les travaux asynchrones ne suffit pas par ellemême à la coconstruction de projets de lecture et à renforcer l'effort interprétatif. Ce résultat incite à rechercher les moyens d'articuler des écrits métalectoraux et métatextuels, inventifs et normés, propositionnels et institutionnalisés en déployant en classe et en ligne un éventail de consignes induisant la réflexion sur le commun et le singulier des expériences esthétiques de lecture.

74 Les usages de forums constituent certainement de bons révélateurs des choix de professeurs pour susciter les interprétations raisonnées. En permettant d'examiner de près les fléchissements réflexifs comme les relances interprétatives, leur étude informe sur les manières de résoudre les difficultés propres à la conduite des lectures analytiques de textes patrimoniaux. Les analyses dont nous avons rendu compte contribuent aussi à caractériser la figure de l'élève lecteur. Dans la mesure où les forums scolaires de lecteurs ouvrent des espaces intermédiaires entre la communauté interprétative des apprentis lecteurs et les interprétations du professeur, ils peuvent apporter des données sur les différentes formes de distanciation que mobilisent les élèves lecteurs pour maintenir une lecture participative et interprétative de textes patrimoniaux.

75 Pour autant, les connaissances des effets d'un usage rationnalisé de forums sur la formation des lecteurs de littérature doivent encore être enrichies. Nous ne disposons pas encore de comparaisons entre des travaux en présentiel et hybrides sur les mêmes textes ou entre des séances menées par des professeurs adoptant un même protocole hybride. Nous savons encore peu de choses sur l'adoption d'une conduite participative en lien avec une recherche de significations chez les élèves les plus éloignés des attentes scolaires. 


\section{BIBLIOGRAPHIE}

AHR, S. (dir.) (2018). Former à la lecture littéraire. Chasseneuil-du-Poitou : Canopé Éd.

BRousSEAU, G. (1998) [1997]. Théorie des situation didactiques. Trad. de l'anglais. Grenoble : La pensée sauvage.

CÉSAIRE, A. (1959) [1947]. Cahier d'un retour au pays natal. Paris : Présence africaine.

COMPAGNON, A. (1998). Le Démon de la théorie. Littérature et sens commun. Paris : Éditions du Seuil.

CITTON, Y. (2007). Lire, interpréter, actualiser. Pourquoi les études littéraires ?. Paris : Amsterdam.

DAVID, J. (2014). «Chloroforme et signification : pourquoi la littérature est-elle si soporifique à

l'école ». Études de lettres 295, p. 19-32. En ligne : https://journals.openedition.org/edl/604.

DUFAYS, J.-L. (2017). « Analyser les pratiques d'enseignement-apprentissage de la lecture des

textes littéraires : quelle modélisation pour quels enjeux ? ». Recherches en éducation 27, p. 11-21. En ligne : https://journals.openedition.org/ree/2907.

DUFAYS, J.-L., GEMENNE, L., \& LEDUR, D. (2015) [1996]. Pour une lecture littéraire, histoire, théories, pistes pour la classe. Louvain-la-Neuve : De Boeck.

ÉluARD, P. (1947). « Notre vie ». In : Le Temps déborde. Paris : Éditions Cahiers d'art.

HUGO, V. (1859). «Sultan Mourad ». In : La légende des Siècles. Paris : Hetzel, p. 253-266. En ligne :

https://fr.wikisource.org/wiki/La_Légende_des_siècles/Sultan_Mourad.

LANGLADE, G. (2008). « Activité fictionnalisante du lecteur et dispositif de l'imaginaire ». In : Roy, M., Brault, M. \& Brehm, S. (dirs). Formation des lecteurs, formation de l'imaginaire. Montréal :

Université du Québec à Montréal, p. 45-65.

LE GOFF, F. \& FOURTANIER, M.-J (2017). Les Formes plurielles des écritures de la réception. Vol. 2, Affects et temporalités. Namur : presses universitaires de Namur.

MACÉ, M. (2011). Façons de lire, manières d'être. Paris : Gallimard.

Ministère de l'Éducation Nationale, de la Jeunesse et des Sport (2008). « Programmes de l'enseignement du français pour les classes de sixième, de cinquième, de quatrième et de troisième du collège ». Bulletin officiel spécial $n^{\circ} 6$ du 28 août 2008. En ligne : https:// www.education.gouv.fr/node/276995.

Ministère de l'Éducation Nationale, de l'Enseignement Supérieur et de la Recherche (2010). « Programme de l'enseignement commun de français en classe de seconde générale et technologique et en classe de première des séries générales et programme de l'enseignement de littérature en classe de première littéraire ». Bulletin officiel spécial $n^{\circ} 9$ du 30 septembre 2010. En ligne : http://www.education.gouv.fr/cid53318/mene1019760a.html.

MOINARD, P. (2017). « Commenter des textes littéraires en collaboration sur des forums et des blogs dans le secondaire » Le Français aujourd'hui 196, p. 71-80. En ligne : https://www.cairn.info/ revue-le-francais-aujourd-hui-2017-1-page-71.html.

MOINARD, P (2019). « Narrations d'hier et d'aujourd'hui sur des forums et des blogs d'apprentis lecteurs. Quels usages des récits pour quels apprentissages? » Pratiques 181-182. En ligne : https:// journals.openedition.org/pratiques/6217. 
RODRIGUEZ, A. (2013) «L'Empathie en poésie lyrique : acte, tension et degrés de lecture ». In : Gefen, A. \& Vouilloux, B. (dirs). Empathie et esthétique. Paris : Hermann, p. 73-101.

SAUVAIRE, M. \& DUfAYS, J.-L. (2020). « Distanciation ». In : Brillant-Rannou, N et al. (dirs). Un

dictionnaire de didactique de la littérature. Paris : H. Champion, p. 236-239.

SCHAEFFER, J.-M. (2015). L'Expérience esthétique. Paris : Gallimard.

\section{NOTES}

1. Voir dans ce numéro l'article de Laetitia Perret, «L'explication de texte et ses avatars : des exercices en tension dans les programmes ».

2. C'est-à-dire des situations associant les travaux en classe et en ligne selon des dosages variables.

3. Dans le système scolaire français, la classe de $3^{e}$ (élèves de $14 / 15$ ans) correspond à la dernière année du collège. Les classes de $2^{\text {nde }}$ et de $1^{\text {re }}$ des lycées généraux et technologiques réunissent des élèves de 15/17 ans.

4. Les programmes du collège de 2008 encore en vigueur en janvier 2016 définissent la lecture analytique "comme une lecture attentive et réfléchie, cherchant à éclairer le sens des textes et à construire chez l'élève des compétences d'analyse et d'interprétation. Elle permet de s'appuyer sur une approche intuitive, sur les réactions spontanées de la classe, pour aller vers une interprétation raisonnée " (Ministère de l'Éducation Nationale, de la Jeunesse et des Sport, 2008, p. 2).

5. Césaire (1959 [1947], p. 59-61).

6. Les programmes du lycée général et technologique de 2010 indiquent que « la lecture analytique vise la construction progressive et précise de la signification d'un texte, quelle qu'en soit l'ampleur; elle consiste donc en un travail d'interprétation que le professeur conduit avec ses élèves, à partir de leurs réactions et de leurs propositions " (Ministère de l'Éducation Nationale, de l'Enseignement Supérieur et de la Recherche, 2010).

7. Les contributions écrites des participants s'affichent automatiquement les unes audessous des autres en commençant par le billet le plus récent.

8. Outre l'affichage automatique des billets en fil, le caractère différé de la communication écrite et l'adressage à tous.

9. Nous ne conservons pas les guillemets pour ce terme et pour le syntagme «lecture participative » dont la définition vient d'être précisée.

10. La « mise en forme du pâtir » distingue pour ce chercheur la discursivité lyrique de la discursivité du récit organisée selon la «logique de l'agir » (Rodriguez, 2013, p. 86). La lecture du lyrique confronte à une "forme affective » (ibid.) qui relie les niveaux sonores, typographiques, métaphoriques $\mathrm{du}$ texte. La conception d'un lecteur affrontant une épreuve d'altération pour entrer dans un monde dépaysant du texte peut être élargie à tous les genres littéraires.

11. On retrouve là le rôle régulateur d'un « calcul hédonique » qui pour J.-M. Schaeffer (2015, p. 318) structure "l'expérience esthétique».

12. Cet équilibre peut aboutir à la reconnaissance par l'apprenti lecteur d'un plaisir lié à la perception de l'étrangeté d'un texte à travers l'expérience de réactions inédites. 
Jérôme David (2014, p. 25) décrit bien cette attitude en définissant « le premier degré de la littérature » comme " un espace d'expérience où le lecteur [...] se livre sans défiance à l'univers d'une œuvre, s'en remet aux formes littéraires comme à des cadres d'expérimentation, s'expose à une pensée autre, à des affects insoupçonnés, à des calibrages inédits de la sensation et de la perception ».

13. Nous indiquons le titre du fil, le prénom de la contributrice suivi du rang du billet dans le fil.

14. Nous ne mentionnons pas les énoncés strictement distanciés (exclusivement centrés sur des procédés) dont les occurrences sont très rares.

15. Le concept de dévolution désigne «l'acte par lequel l'enseignant fait accepter à l'élève la responsabilité d'une situation d'apprentissage ou d'un problème et accepte lui-même les conséquences de ce transfert » (Brousseau, 1998 [1997], p. 303).

16. La consigne invite à simuler un dialogue entre adolescents lecteurs sous le regard professoral et pour répondre à des attentes scolaires.

17. « Le porc, dont un frisson agitait les vertèbres,

Râlait, triste, épuisé, morne ; et le padischah

De cet être difforme et sanglant s'approcha,

Comme on s'arrête au bord d'un gouffre qui se creuse ;

Mourad pencha son front vers la bête lépreuse,

Puis la poussa du pied dans l'ombre du chemin, » (Hugo, 1859, III)

18. «Et Mourad entendit une voix qui disait : [...]

Mais tu viens d'avoir, monstre, un éclair de pitié [...]

Un pourceau secouru pèse un monde opprimé ;

Viens! le ciel s'offre, avec ses étoiles sans nombre.

En frémissant de joie, à l'évadé de l'ombre ! (Hugo, 1859, V)

19. Dont les influences relatives nous semblent impossibles à spécifier.

20. Seul le billet 22 est centré sur les possibles significations du texte. Il est difficile d'évaluer l'influence spécifique de la situation de communication en ligne dans l'effet d'écho entre les billets. Une comparaison du fil avec 23 productions sur papier d'une autre classe de seconde en réponse à la même consigne montre que les copies présentent moins de retours sur la lecture participative que les billets mais plus d'hypothèses de significations. Il est possible que les élèves envisagent le travail sur copies comme un exercice plus scolaire exigeant l'examen des significations du texte étudié.

21. En entretien, elle expose son intention de faire émerger en classe et en ligne des " points d'achoppement » à travers les jugements axiologiques et esthétiques spontanés des élèves.

22. La barre oblique code une brève pause.

23. Pour la déclarante, la diversité est relative : les «autres " sont des semblables au sein du collectif que forme la classe de première technologique particulièrement soudée.

24. Et qui peuvent prendre une certaine influence dans la mesure où elles sont publiées.

25. La grille d'analyse des captations employée par les membres de l'équipe Pelas prévoit le repérage de cinq «moments » structurant le synopsis des séances de lecture 
analytique : l'accueil des élèves, la (re)mobilisation des élèves lecteurs, la gestion de la réception, l'émergence du projet de lecture, le déroulé des activités et l'institutionnalisation.

26. Le motif du jet d'objets est développé dans le verset suivant dont l'inachèvement a suscité, en classe, quelques interrogations : "On lui jetait des pierres, des bouts de ferrailles, des tessons de bouteille, mais ni ces pierres, ni cette ferraille, ni ces bouteilles... ».

27. L'élève fait allusion au verset suivant: "Le négrier craque de toute part ... Son ventre se convulse et résonne ... L'affreux ténia de sa cargaison ronge les boyaux fétides de l'étrange nourrisson des mers! »

28. L'opposition horizontalité/verticalité de la communication n'équivaut pas à la distinction entre échanges scolaires et non scolaires. Différentes formes d'horizontalité (relative) en contexte scolaire peuvent se manifester en classe comme en ligne quand les élèves sont conduits à rebondir directement sur des interventions des autres (par exemple lors de travaux en équipe).

29. Au sens premier : les réactions évoquées par écrit deviennent des objets de travail et de discussion.

30. Les relectures des billets sont donc étayées mais nous ne disposons pas de traces des régulations professorales au cours de ces séances.

31. Le sujet de cet entrainement à l'épreuve écrite du baccalauréat de français indique des axes de lecture du commentaire littéraire.

\section{RÉSUMÉS}

L'article interroge les modalités d'une co-élaboration du questionnement sur des textes littéraires et leurs effets sur la formation des lecteurs lors de trois séances incluant des temps d'échanges sur des forums dans des classes de $3^{\mathrm{e}}, 2^{\text {nde }}$ et $1^{\text {re }}$. Le cadre théorique de l'étude intègre la notion d'activités fictionnalisantes du sujet-lecteur (Langlade, 2008) au paradigme dialectique de la «lecture littéraire» (Dufays, Gemenne \& Ledur, 2015 [1996]) et précise la notion de participation du lecteur. La méthodologie d'analyse permet de suivre les évolutions des interactions entre élèves lecteurs en ligne et en classe en lien avec les consignes des professeurs. Les analyses dont l'article rend compte montrent que le partage des billets de lecteurs entraine des retours variés sur les expériences de réception. Elles informent sur les conditions d'utilisation de forums favorables à l'effort interprétatif et permettent d'identifier des facteurs limitant la réflexion collaborative des élèves lecteurs.

The article questions the modalities of co-elaboration of the questioning on literary texts and their effects on the training of readers during three sessions including exchange times on student forums in years 10,11 and $12 / 9^{\text {th }}, 10^{\text {th }}$ and $11^{\text {th }}$ grades. The theoretical framework of the study incorporates the notion of fictional activities of the reader subject (Langlade, 2008) into the dialectical paradigm of "literary reading" (Dufays, Gemenne \& Ledur, 2015 [1996]). It also clarifies the notion of reader's participation. The methodology of analysis makes it possible to follow the evolution of interactions between students readers online and in class in connection with the 
instructions of the teachers. The analyses reported in the article show that the sharing of readers' tickets leads to varied feedback on receiving experiences. The results obtained inform on the conditions of use of forums favorable to the interpretative effort. They also identify factors that limit the collaborative thinking by student readers

INDEX

Mots-clés : approches analytiques, lecture littéraire, enseignements hybrides, forums

Keywords : analytical approaches, literary reading, blended learning, forums

\section{AUTEUR}

PIERRE MOINARD

Université de Poitiers, Laboratoire FoReLLIS, F-86073 Poitiers, France 